\title{
Exact Travelling Wave Solutions of Two Nonlinear Schrödinger Equations by Using Two Methods
}

\author{
Qingmei Zhang, Mei Xiong, Longwei Chen \\ College of Statistics and Mathematics, Yunnan University of Finance and Economics, Kunming, Yunnan, China \\ Email: 1477273038@qq.com,tc715@sina.com,clwxmff@163.com
}

How to cite this paper: Zhang, Q.M., Xiong, M. and Chen, L.W. (2019) Exact Travelling Wave Solutions of Two Nonlinear Schrödinger Equations by Using Two Methods. Journal of Applied Mathematics and Physics, 7, 3101-3115.

https://doi.org/10.4236/jamp.2019.712218

Received: November 10, 2019

Accepted: December 15, 2019

Published: December 18, 2019

Copyright $\odot 2019$ by author(s) and Scientific Research Publishing Inc. This work is licensed under the Creative Commons Attribution International License (CC BY 4.0).

http://creativecommons.org/licenses/by/4.0/

\begin{abstract}
The special kind of $\left(G^{\prime} / G\right)$-expansion method and the new mapping method are easy and significant mathematical methods. In this paper, exact travelling wave solutions of the higher order dispersive Cubic-quintic nonlinear Schrödinger equation and the generalized nonlinear Schrödinger equation are studied by using the two methods. Finally, the solitary wave solutions, singular soliton solutions, bright and dark soliton solutions and periodic solutions of the two nonlinear Schrödinger equations are obtained. The results show that this method is effective for solving exact solutions of nonlinear partial differential equations.
\end{abstract}

\section{Keywords}

The Special Kind of $\left(G^{\prime} / G\right)$-Expansion Method, the New Mapping Method, the Partial Differential Equations, the Exact Travelling Wave Solutions

\section{Introduction}

The nonlinear PDE is an important model for describing the problems of Nonlinear phenomenon, such as hydrodynamics, plasma physics, chemical dynamics, photobiology, solid physics, Marine and atmospheric phenomena, and so on. It can be seen from these fields that the travelling wave solutions of nonlinear evolution equations play an important role in the study. In order to find the exact solutions of nonlinear partial differential Equations (PDEs), pioneers presented the following these methods, such as the first integral method [1], Jacobi elliptic function expansion method [2], F expansion method [3], exp-function method [4], the Kudryashov method [5], the improved $\left(G^{\prime} / G\right)$-expansion method [6], the tanh-coth method [7], tanh-sech method [8], projective Riccati equation 
method [9], Kudryashov method [10], sine-cosine method [11], Hirota bilinear method [12], bifurcation theory method of dynamic systems [13] and so on.

In this article, we consider the higher order dispersive Cubic-quintic nonlinear Schrödinger Equation (NLSE), see [14] and the generalized nonlinear Schrödinger Equation (GNLSE), see [15]:

$$
i q_{Z}-\frac{\beta_{2}}{2} q_{t t}+\gamma_{1}|q|^{2} q-i \frac{\beta_{3}}{6} q_{t t t}-\frac{\beta_{4}}{24} q_{t t t t}+\gamma_{2}|q|^{4} q=0
$$

and

$$
i u_{t}-r_{2} u_{x x}+c_{3}|u|^{2} u=i\left[\left(s_{0}+s_{2}|u|^{2}\right) u\right]_{x}-c_{5}|u|^{4} u .
$$

where $\beta_{2}, \beta_{3}, \beta_{4}, \gamma_{1}, \gamma_{2}, r_{2}, c_{3}, c_{5}, s_{0}, s_{2}$ are real constants. $q, u$ are complex functions.

In 2014, Kudryashov [16] substantiated that the $\left(G^{\prime} / G\right)$-expansion method together with the linear ordinary differential equation

$G^{\prime \prime}-\lambda G^{\prime}-\mu G=0, \lambda, \mu \in \mathfrak{R}$ is identical to the well-known tanh-method. Furthermore, In 2014, Alam and Akbar [17] [18] researched extremely significant extension of the $\left(G^{\prime} / G\right)$-expansion method to receive exact travelling wave solutions of nonlinear evolution equations, For the new mapping method, scholars introduced this method, see [19] [20] [21], and gave the specific solving process for nonlinear PDE.

For the higher order dispersive Cubic-quintic NLSE, In 2017, Zayed and Nowehy [22] incorporated the solution Ansatz method with the Jacobi elliptic equation method to obtain several integrations denoted Jacobi elliptic function of the equation. In 2017, Arshad, sedawy and Lu [23] used an improved direct algebraic extension method to present bright and dark wave solutions and soliton wave solutions of higher order dispersive Cubic-quintic NLSEs. In addition, there is an amount of paper [24] [25] [26] where the various types of the equation are studied. For the GNLSE, In 2010, Geng and Li by using the dynamic system method and bifurcation theory, studies the travelling wave solution of the GNLSE and high order dispersion NLSE. the solitary wave solutions, kink and reverse kink wave solutions and periodic wave solutions are obtained. In 2007, Huang, Li and Zhang [27] through the study a class of nonlinear term six times of first order nonlinear ODE and applies it to the GNLSE. New accurate traveling wave solutions, such as light and dark isolated wave solutions, triangular periodic wave solutions and singular solutions are obtained. In addition, this GNLSE was studied, see [28] [29] [30].

The rest of the article is organized as follows: Section 2, we mainly describe the basic idea of the special kind of $\left(G^{\prime} / G\right)$-expansion method and the new mapping method briefly. In Section 3 and 4, we use these two methods to solve two NLSEs in detail. Some conclusions are drawn in Section 4.

\section{Introduction of Two Methods}

Method 1: The special kind of $\left(G^{\prime} / G\right)$-expansion method. 
Consider the general nonlinear PDE of the form:

$$
P\left(u, u_{t}, u_{x}, u_{x x}, u_{t t}, u_{x t}, u_{x x x}, \cdots\right)=0 .
$$

where $P$ is a polynomial in its arguments.

In order to transform the Equation (3) into an ODE, we suppose that

$$
u(x, t)=u(\xi), \xi=x-c t .
$$

where $\mathrm{c}$ is a constant, then

$$
\frac{\partial}{\partial t}(\cdot)=-c \frac{\partial}{\partial \xi}(\cdot), \frac{\partial}{\partial x}(\cdot)=\frac{\partial}{\partial \xi}(\cdot), \frac{\partial^{2}}{\partial t^{2}}(\cdot)=c^{2} \frac{\partial^{2}}{\partial \xi^{2}}(\cdot), \cdots .
$$

Step 1: According to above supposing, the Equation (3) has the following nonlinear ODE form:

$$
Q\left(u, u_{\xi}, u_{\xi \xi}, \cdots\right)=0 .
$$

where the subscript denotes the derivation with respect to $\xi$.

Step 2: Suppose that the Equation (6) has non-integer balance number $N$. the solution of the Equation (6) can be written in the following special form, see [31] [32] [33]:

$$
u(\xi)=\Omega\left(\frac{G^{\prime}}{G}\right)^{N}
$$

where $G(\xi)$ satisfies the linear ODE:

$$
G^{\prime \prime}(\xi)+\lambda G^{\prime}(\xi)+\mu G(\xi)=0 .
$$

Step 3: Firstly, determining the balance number $\mathrm{N}$ by balancing the high order derivative and the highest power of the nonlinear term in Equation (6).

Step 4: Then, substituting the Equations (7) and (8) into the Equation (6), and make the coefficients of $\left[\frac{G^{\prime}(\xi)}{G(\xi)}\right]$ all zero, and get a set of algebraic equations, which can be solved by Maple software to find $\Omega, \lambda, \mu, c$.

Step 5: Finally by solving Equation (8) for $\left[\frac{G^{\prime}(\xi)}{G(\xi)}\right]$ ratio, the Equation (3) exact solutions are obtained.

Method 2: The new mapping method.

Step 1: We suppose that the Equation (6) has the formal solution:

$$
u(\xi)=F(\varphi(\xi))
$$

where $F$ is an appropriate variable transformation, and $\varphi(\xi)$ satisfies the following equation:

$$
\varphi^{\prime 2}(\xi)=\delta+\alpha \varphi^{2}(\xi)+\frac{\beta}{2} \varphi^{4}(\xi)+\frac{\gamma}{3} \varphi^{6}(\xi)
$$

where $\delta, \alpha, \beta, \gamma$ are arbitrary constant to be determined.

Step 2: It can be seen from the solution [34] that the Equation (9) has the formal solutions with $\gamma \neq 0$. 


$$
\begin{aligned}
& \varphi_{1}(\xi)=4 \sqrt{\frac{-\alpha \tanh ^{2}\left(\epsilon \sqrt{-\frac{\alpha}{3}} \xi\right)}{3 \beta\left[3+\tanh ^{2}\left(\epsilon \sqrt{-\frac{\alpha}{3}} \xi\right)\right]}}, \alpha<0, \beta>0, \gamma=\frac{3 \beta^{2}}{16 \alpha}, \delta=\frac{16 \alpha^{2}}{27 \beta} . \\
& \varphi_{2}(\xi)=4 \sqrt{\frac{-\alpha \operatorname{coth}^{2}\left(\epsilon \sqrt{-\frac{\alpha}{3}} \xi\right)}{3 \beta\left[3+\operatorname{coth}^{2}\left(\epsilon \sqrt{-\frac{\alpha}{3}} \xi\right)\right]}}, \alpha<0, \beta>0, \gamma=\frac{3 \beta^{2}}{16 \alpha}, \delta=\frac{16 \alpha^{2}}{27 \beta} . \\
& \varphi_{3}(\xi)=4 \sqrt{\frac{\alpha \tan ^{2}\left(\epsilon \sqrt{\frac{\alpha}{3}} \xi\right)}{3 \beta\left[3-\tan ^{2}\left(\epsilon \sqrt{\frac{\alpha}{3}} \xi\right)\right]}}, \alpha>0, \beta<0, \gamma=\frac{3 \beta^{2}}{16 \alpha}, \delta=\frac{16 \alpha^{2}}{27 \beta} . \\
& \varphi_{4}(\xi)=4 \sqrt{\frac{\alpha \cot ^{2}\left(\epsilon \sqrt{\frac{\alpha}{3}} \xi\right)}{3 \beta\left[3-\cot ^{2}\left(\epsilon \sqrt{\frac{\alpha}{3}} \xi\right)\right]}}, \alpha>0, \beta<0, \gamma=\frac{3 \beta^{2}}{16 \alpha}, \delta=\frac{16 \alpha^{2}}{27 \beta} . \\
& \varphi_{5}(\xi)=\sqrt{\frac{-2 \alpha}{\beta}[1+\tanh (\epsilon \sqrt{\alpha} \xi)]}, \alpha>0, \gamma=\frac{3 \beta^{2}}{16 \alpha}, \delta=0 . \\
& \varphi_{6}(\xi)=\sqrt{\frac{-2 \alpha}{\beta}[1+\operatorname{coth}(\epsilon \sqrt{\alpha} \xi)]}, \alpha>0, \gamma=\frac{3 \beta^{2}}{16 \alpha}, \delta=0 . \\
& \varphi_{7}(\xi)=\sqrt{\frac{-6 \alpha \beta \operatorname{sech}^{2}(\sqrt{\alpha} \xi)}{3 \beta^{2}-4 \alpha \gamma[1+\epsilon \tanh (\sqrt{\alpha} \xi)]^{2}}}, \alpha>0, \delta=0 \text {. } \\
& \varphi_{8}(\xi)=\sqrt{\frac{6 \alpha \beta \operatorname{csch}^{2}(\sqrt{\alpha} \xi)}{3 \beta^{2}-4 \alpha \gamma[1+\epsilon \operatorname{coth}(\sqrt{\alpha} \xi)]^{2}}}, \alpha>0, \delta=0 \text {. } \\
& \varphi_{9}(\xi)=\sqrt{\frac{-6 \alpha \operatorname{sech}^{2}(\sqrt{\alpha} \xi)}{3 \beta+4 \epsilon \sqrt{3 \alpha \gamma} \tanh (\sqrt{\alpha} \xi)}}, \alpha>0, \gamma>0, \delta=0 . \\
& \varphi_{10}(\xi)=\sqrt{\frac{6 \alpha \operatorname{csch}^{2}(\sqrt{\alpha} \xi)}{3 \beta+4 \epsilon \sqrt{3 \alpha \gamma} \operatorname{coth}(\sqrt{\alpha} \xi)}}, \alpha>0, \gamma>0, \delta=0 \text {. } \\
& \varphi_{11}(\xi)=\sqrt{\frac{-6 \alpha \operatorname{sech}^{2}(\sqrt{-\alpha} \xi)}{3 \beta+4 \epsilon \sqrt{-3 \alpha \gamma} \tanh (\sqrt{-\alpha} \xi)}}, \alpha<0, \gamma>0, \delta=0 \text {. } \\
& \varphi_{12}(\xi)=\sqrt{\frac{-6 \alpha \operatorname{csch}^{2}(\sqrt{-\alpha} \xi)}{3 \beta+4 \epsilon \sqrt{-3 \alpha \gamma} \operatorname{coth}(\sqrt{-\alpha} \xi)}}, \alpha<0, \gamma>0, \delta=0 \text {. }
\end{aligned}
$$




$$
\begin{aligned}
& \varphi_{13}(\xi)=2 \sqrt{\frac{3 \alpha}{\epsilon \sqrt{Q} \cosh (2 \sqrt{\alpha} \xi)-3 \beta}}, \alpha>0, Q>0, \delta=0 . \\
& \varphi_{14}(\xi)=2 \sqrt{\frac{3 \alpha \epsilon \operatorname{sech}(2 \sqrt{\alpha} \xi)}{\sqrt{Q}-3 \epsilon \beta \operatorname{sech}(2 \sqrt{\alpha} \xi)}}, \alpha>0, Q>0, \delta=0 . \\
& \varphi_{15}(\xi)=2 \sqrt{\frac{3 \alpha \operatorname{sech}^{2}(\epsilon \sqrt{\alpha} \xi)}{2 \sqrt{Q}-(\sqrt{Q}+3 \beta) \operatorname{sech}^{2}(\epsilon \sqrt{\alpha} \xi)}}, \alpha>0, \beta<0, \gamma<0, Q>0, \delta=0 \text {. } \\
& \varphi_{16}(\xi)=2 \sqrt{\frac{3 \alpha \operatorname{csch}^{2}(\epsilon \sqrt{\alpha} \xi)}{2 \sqrt{Q}+(\sqrt{Q}-3 \beta) \operatorname{csch}^{2}(\epsilon \sqrt{\alpha} \xi)}}, \alpha>0, \beta<0, \gamma<0, Q>0, \delta=0 \text {. } \\
& \varphi_{17}(\xi)=2 \sqrt{\frac{-3 \alpha \operatorname{sech}^{2}(\epsilon \sqrt{-\alpha} \xi)}{2 \sqrt{Q}-(\sqrt{Q}-3 \beta) \operatorname{sech}^{2}(\epsilon \sqrt{-\alpha} \xi)}}, \alpha<0, \beta>0, \gamma<0, Q>0, \delta=0 \text {. } \\
& \varphi_{18}(\xi)=2 \sqrt{\frac{-3 \alpha \operatorname{csch}^{2}(\epsilon \sqrt{-\alpha} \xi)}{2 \sqrt{Q}-(\sqrt{Q}+3 \beta) \operatorname{csch}^{2}(\epsilon \sqrt{-\alpha} \xi)}}, \alpha<0, \beta>0, \gamma<0, Q>0, \delta=0 . \\
& \varphi_{19}(\xi)=2 \sqrt{\frac{3 \alpha}{\epsilon \sqrt{Q} \cos (2 \sqrt{-\alpha} \xi)-3 \beta}}, \alpha<0, Q>0, \delta=0 . \\
& \varphi_{20}(\xi)=2 \sqrt{\frac{3 \alpha}{\epsilon \sqrt{Q} \sin (\epsilon \sqrt{-\alpha} \xi)-3 \beta}}, \alpha<0, Q>0, \delta=0 . \\
& \varphi_{21}(\xi)=2 \sqrt{\frac{3 \alpha \epsilon \sec (2 \sqrt{-\alpha} \xi)}{\sqrt{Q}-3 \epsilon \beta \sec (2 \sqrt{-\alpha} \xi)}}, \alpha<0, Q>0, \delta=0 \text {. } \\
& \varphi_{22}(\xi)=2 \sqrt{\frac{3 \alpha \epsilon \csc (2 \sqrt{-\alpha} \xi)}{\sqrt{Q}-3 \epsilon \beta \csc (2 \sqrt{-\alpha} \xi)}}, \alpha<0, Q>0, \delta=0 \text {. } \\
& \varphi_{23}(\xi)=2 \sqrt{\frac{3 \alpha}{\epsilon \sqrt{-Q} \cos (2 \sqrt{\alpha} \xi)-3 \beta}}, \alpha<0, Q>0, \delta=0 .
\end{aligned}
$$

where $Q=9 \beta^{2}-48 \alpha \gamma, \epsilon= \pm 1$.

Step 3: Substituting the solutions (11) - (33) into Equation (9) to get the exact solutions of Equation (3).

\section{Application of the Special Kind of $\left(G^{\prime} / G\right)$-Expansion \\ Method}

In this section, we apply the special kind of $\left(G^{\prime} / G\right)$-expansion method to solve the two higher order NLSE.

Firstly, for Equation (1). We suppose that the Equation (1) has the following solution: 


$$
q(Z, t)=\varphi(\xi) \mathrm{e}^{i(\lambda Z-w t)} .
$$

where $\xi=p Z-v t$. And $p, v, \lambda, w$ are real parameters, $\varphi(\xi)$ is real function.

Substituting Equation (34) into Equation (1), and putting imaginary and real part are zero respectively:

$$
\begin{aligned}
& \left(\beta_{3}-\beta_{4} w\right) v^{3} \varphi^{\prime \prime \prime}+\left(6 p-6 \beta_{2} v w-3 \beta_{3} v w^{2}+\beta_{4} v w^{3}\right) \varphi^{\prime}=0, \\
& \beta_{4} v^{4} \varphi^{\prime \prime \prime \prime}+\left(-6 \beta_{4} v^{2} w^{2}+12 \beta_{3} v^{2} w+12 \beta_{2} v^{2}\right) \varphi^{\prime \prime} \\
& +\left(-12 \beta_{2} w^{2}+24 \lambda-4 \beta_{3} w^{3}+\beta_{4} w^{4}\right) \varphi-24 \gamma_{1} \varphi^{3}-24 \gamma_{2} \varphi^{5}=0 .
\end{aligned}
$$

Differentiating Equation (35) once, and substituting the resultant equation into Equation (36), we let $k_{1}=\left(\beta_{3}-\beta_{4} w\right) v^{3}, k_{2}=6 p-6 \beta_{2} v w-3 \beta_{3} v w^{2}+\beta_{4} v w^{3}$, $k_{3}=-6 \beta_{4} v^{2} w^{2}+12 \beta_{3} v^{2} w+12 \beta_{2} v^{2} \quad$ and $\quad k_{4}=-12 \beta_{2} w^{2}+24 \lambda-4 \beta_{3} w^{3}+\beta_{4} w^{4}$. then we can get

$$
\varphi^{\prime \prime}-\frac{24 \gamma_{2} k_{1}}{k_{1} k_{3}-\beta_{4} v^{4} k_{2}} \varphi^{5}-\frac{24 \gamma_{1} k_{1}}{k_{1} k_{3}-\beta_{4} v^{4} k_{2}} \varphi^{3}+\frac{k_{4} k_{1}}{k_{1} k_{3}-\beta_{4} v^{4} k_{2}} \varphi=0 .
$$

Multiplying (37) by $\varphi^{\prime}(\xi)$ and integrating once with respect to $\xi$, we have the auxiliary equation:

$$
\varphi^{\prime 2}(\xi)=\sigma_{0}+\sigma_{2} \varphi^{2}(\xi)+\sigma_{4} \varphi^{4}(\xi)+\sigma_{6} \varphi^{6}(\xi),
$$

where $\sigma_{0}=2 \epsilon_{1}, \quad \sigma_{2}=-\frac{k_{4} k_{1}}{k_{1} k_{3}-\beta_{4} v^{4} k_{2}}, \quad \sigma_{4}=\frac{12 \gamma_{1} k_{1}}{k_{1} k_{3}-\beta_{4} v^{4} k_{2}}, \quad \sigma_{6}=\frac{8 \gamma_{2} k_{1}}{k_{1} k_{3}-\beta_{4} v^{4} k_{2}}$, and $\epsilon_{1}$ is the integral constant.

Secondly, for Equation (2), We suppose that the Equation (2) has the following solution:

$$
u(x, t)=\varphi(\xi) \mathrm{e}^{i(x-\omega t)} .
$$

where $\xi=x-\lambda t$. And $\lambda, \omega$ are real parameters.

Substituting Equation (39) into Equation (2), and putting real and imaginary part are zero respectively:

$$
\begin{gathered}
\left(2 \gamma_{2}-\lambda-s_{0}\right) \varphi^{\prime}-3 s_{2} \varphi^{2} \varphi^{\prime}=0 \\
r_{2} \varphi^{\prime \prime}+\left(\omega+s_{0}-\gamma_{2}\right) \varphi+\left(c_{3}+s_{2}\right) \varphi^{3}+c_{5} \varphi^{5}=0 .
\end{gathered}
$$

Integrating Equation (40) once, and substituting the resultant equation into Equation (41),

$$
\varphi^{\prime \prime}+\frac{\omega+\gamma_{2}-\lambda}{r_{2}} \varphi+\frac{c_{3}}{r_{2}} \varphi^{3}+\frac{c_{5}}{r_{2}} \varphi^{5}=0
$$

Multiplying (42) by $\varphi^{\prime}(\xi)$ and integrating once with respect to $\xi$, we have the auxiliary equation:

$$
\varphi^{\prime 2}(\xi)=\sigma_{0}+\sigma_{2} \varphi^{2}(\xi)+\sigma_{4} \varphi^{4}(\xi)+\sigma_{6} \varphi^{6}(\xi),
$$

If we make again $\sigma_{0}=2 \epsilon_{1}, \sigma_{2}=-\frac{\omega+\gamma_{2}-\lambda}{r_{2}}, \sigma_{4}=-\frac{c_{3}}{2 r_{2}}, \sigma_{6}=-\frac{c_{5}}{3 r_{2}}$, and $\epsilon_{1}$ is the integral constant. 
Then, Observed Equations (38) and (43), to find the exact solutions of the them, we only need to discuss one of these equations. Next, we will give the solving process of the Equation (38).

Now we will use the special $\left(G^{\prime} / G\right)$ expansion method to solve Equation (38), therefore, according to Section 2 as follows: Balancing $\varphi^{\prime 2}$ with $\varphi^{6}$ yields $N=\frac{1}{2}$, then Equation (38) solution has the following solution:

$$
\psi(\xi)=\Omega\left(\frac{G^{\prime}(\xi)}{G(\xi)}\right)^{\frac{1}{2}} .
$$

where $\Omega$ is the constant to be determined, $G(\xi)$ satisfy Equation (8), Substituting Equation (44) and Equation (8) into Equation (38), we get the following equation:

$$
\begin{aligned}
& \left\{-\frac{1}{2} \Omega \lambda\left(\frac{G^{\prime}}{G}\right)^{\frac{1}{2}}-\frac{1}{2} \Omega \mu\left(\frac{G^{\prime}}{G}\right)^{-\frac{1}{2}}-\frac{1}{2} \Omega\left(\frac{G^{\prime}}{G}\right)^{\frac{3}{2}}\right\}^{2} \\
& =\sigma_{0}+\sigma_{2} \Omega^{2}\left(\frac{G^{\prime}}{G}\right)+\sigma_{4} \Omega^{4}\left(\frac{G^{\prime}}{G}\right)^{2}+\sigma_{6} \Omega^{6}\left(\frac{G^{\prime}}{G}\right)^{3} .
\end{aligned}
$$

By comparing the power coefficient of the Equation (45), we can get the algebraic equations:

$$
\left\{\begin{array}{l}
\left(\frac{G^{\prime}}{G}\right)^{0}: \frac{1}{2} \Omega^{2} \lambda \mu=\sigma_{0} \\
\left(\frac{G^{\prime}}{G}\right)^{1}: \frac{1}{4} \Omega^{2} \lambda^{2}+\frac{1}{2} \Omega^{2} \mu=\sigma_{2} \Omega^{2} \\
\left(\frac{G^{\prime}}{G}\right)^{-1}: \frac{1}{4} \Omega^{2} \mu^{2}=0 \\
\left(\frac{G^{\prime}}{G}\right)^{3}: \frac{1}{4} \Omega^{2}=\sigma_{6} \Omega^{6} \\
\left(\frac{G^{\prime}}{G}\right)^{2}: \frac{1}{2} \Omega^{2} \lambda=\sigma_{4} \Omega^{4}
\end{array}\right.
$$

Using Maple solving them, we can obtain the following coefficients:

$$
\mu=0, \sigma_{0}=0, \sigma_{2}=\frac{1}{4} \lambda^{2}, \Omega^{2}=\frac{\lambda}{2 \sigma_{4}}, \lambda=\frac{\sigma_{4}^{2}}{\sigma_{6}} .
$$

Then, the new exact solution of Equation (38) is:

$$
\varphi(\xi)=\left[\frac{3 \gamma_{1}}{4 \gamma_{2}}\left(\frac{\Omega_{1} \exp (-\lambda \xi)}{\Omega_{0}+\Omega_{1} \exp (-\lambda \xi)}\right)\right]^{\frac{1}{2}} .
$$

where, $\Omega_{0}$ and $\Omega_{1}$ are arbitrary integral constants and $\xi=p Z-v t$.

In particular, If we choose $\Omega_{0}=\Omega_{1}=1$, then we can get the dark soliton solutions of Equation (38): 


$$
\varphi(\xi)=\left[\frac{3 \gamma_{1}}{4 \gamma_{2}}\left(1 \pm \tanh \left(\xi \sqrt{\frac{-k_{1} k_{4}}{k_{1} k_{3}-\beta_{4} v^{4} k_{2}}}\right)\right)\right]^{\frac{1}{2}} .
$$

If we choose $\Omega_{0}=\Omega_{1}=-1$, then we can get the singular soliton solutions Equation (38):

$$
\varphi(\xi)=\left[\frac{3 \gamma_{1}}{4 \gamma_{2}}\left(1 \pm \operatorname{coth}\left(\xi \sqrt{\frac{-k_{1} k_{4}}{k_{1} k_{3}-\beta_{4} v^{4} k_{2}}}\right)\right)\right]^{\frac{1}{2}} .
$$

where satisfy the constraint condition: $\gamma_{1} \gamma_{2}>0, \frac{k_{1} k_{4}}{k_{1} k_{3}-\beta_{4} v^{4} k_{2}}<0$, and $\xi=p Z-v t$.

\section{Application of the New Mapping Method}

In this section, we apply the new mapping method to solve the two higher order NLSE.

Firstly, we rewrite the Equation (38) to take the following form:

$$
\varphi^{\prime 2}(\xi)=\delta_{0}+\alpha \varphi^{2}(\xi)+\frac{\beta}{2} \varphi^{4}(\xi)+\frac{\gamma}{3} \varphi^{6}(\xi)
$$

where $\delta_{0}=2 \epsilon_{1}, \quad \alpha=-\frac{k_{4} k_{1}}{k_{1} k_{3}-\beta_{4} v^{4} k_{2}}, \quad \beta=\frac{24 \gamma_{1} k_{1}}{k_{1} k_{3}-\beta_{4} v^{4} k_{2}}, \quad \gamma=\frac{24 \gamma_{2} k_{1}}{k_{1} k_{3}-\beta_{4} v^{4} k_{2}}, \quad \epsilon_{1}$ is the integral constant.

According to Section 2, the method is applied to Equation (51), then the solution of Equation (51) is obtained as follows:

1) If $\alpha<0, \beta>0, \gamma=\frac{3 \beta^{2}}{16 \alpha}, \delta=\frac{16 \alpha^{2}}{27 \beta}$, Then, we derive from Equation and Equation (12) that Equation (51) has the solitary wave solutions:

$$
\varphi(\xi)=\left\{\frac{2 k_{4}}{9 \gamma_{1}}\left[\frac{\tanh ^{2}\left(\xi \sqrt{-\frac{k_{1} k_{4}}{3\left(k_{1} k_{3}-\beta_{4} v^{4} k_{2}\right)}}\right)}{3+\tanh ^{2}\left(\xi \sqrt{-\frac{k_{1} k_{4}}{3\left(k_{1} k_{3}-\beta_{4} v^{4} k_{2}\right)}}\right)}\right]\right\}^{\frac{1}{2}},
$$

and

$$
\varphi(\xi)=\left\{\frac{2 k_{4}}{9 \gamma_{1}}\left[\frac{\operatorname{coth}^{2}\left(\xi \sqrt{-\frac{k_{1} k_{4}}{3\left(k_{1} k_{3}-\beta_{4} v^{4} k_{2}\right)}}\right)}{3+\operatorname{coth}^{2}\left(\xi \sqrt{-\frac{k_{1} k_{4}}{3\left(k_{1} k_{3}-\beta_{4} v^{4} k_{2}\right)}}\right)}\right]\right\}^{\frac{1}{2}},
$$

where satisfy the constraint condition: $\gamma_{1} k_{4}>0, \frac{k_{1} k_{4}}{k_{1} k_{3}-\beta_{4} v^{4} k_{2}}<0, \epsilon_{1}=\frac{16 \alpha^{2}}{54 \beta}$, and $\xi=p Z-v t$. 
2) If $\alpha>0, \beta<0, \gamma=\frac{3 \beta^{2}}{16 \alpha}, \delta=\frac{16 \alpha^{2}}{27 \beta}$, Then, we derive from Equation and Equation (14) that Equation (51) has the periodic solutions:

$$
\varphi(\xi)=\left\{-\frac{2 k_{4}}{9 \gamma_{1}}\left[\frac{\tan ^{2}\left(\xi \sqrt{\frac{k_{1} k_{4}}{3\left(k_{1} k_{3}-\beta_{4} v^{4} k_{2}\right)}}\right)}{3-\tanh ^{2}\left(\xi \sqrt{\frac{k_{1} k_{4}}{3\left(k_{1} k_{3}-\beta_{4} v^{4} k_{2}\right)}}\right)}\right]\right\}^{\frac{1}{2}},
$$

and

$$
\varphi(\xi)=\left\{-\frac{2 k_{4}}{9 \gamma_{1}}\left[\frac{\cot ^{2}\left(\xi \sqrt{\frac{k_{1} k_{4}}{3\left(k_{1} k_{3}-\beta_{4} v^{4} k_{2}\right)}}\right)}{3-\cot ^{2}\left(\xi \sqrt{\frac{k_{1} k_{4}}{3\left(k_{1} k_{3}-\beta_{4} v^{4} k_{2}\right)}}\right)}\right]\right\}^{\frac{1}{2}},
$$

where satisfy the constraint condition: $\gamma_{1} k_{4}<0, \frac{k_{1} k_{4}}{k_{1} k_{3}-\beta_{4} v^{4} k_{2}}>0, \epsilon_{1}=\frac{16 \alpha^{2}}{54 \beta}$, and $\xi=p Z-v t$.

3) If $\alpha>0, \gamma=\frac{3 \beta^{2}}{16 \alpha}, \delta=0$, Then, we derive from Equation (15) and Equation (16) that Equation (51) has the Dark soliton solutions:

$$
\varphi(\xi)=\left[\frac{k_{4}}{12 \gamma_{1}}\left(1 \pm \tanh \left(\xi \sqrt{\frac{k_{1} k_{4}}{k_{1} k_{3}-\beta_{4} v^{4} k_{2}}}\right)\right)^{\frac{1}{2}} .\right.
$$

and the singular soliton solutions:

$$
\varphi(\xi)=\left[\frac{k_{4}}{12 \gamma_{1}}\left(1 \pm \operatorname{coth}\left(\xi \sqrt{\frac{k_{1} k_{4}}{k_{1} k_{3}-\beta_{4} v^{4} k_{2}}}\right)\right)^{\frac{1}{2}} .\right.
$$

where satisfy the constraint condition: $\gamma_{1} k_{4}>0, \frac{k_{1} k_{4}}{k_{1} k_{3}-\beta_{4} v^{4} k_{2}}>0, \epsilon_{1}=0$, and $\xi=p Z-v t$.

4) If $\alpha>0, \delta=0$, Then, we derive from Equation (17) and Equation (18) that Equation (51) has the solitary wave solutions:

$$
\varphi(\xi)=\left\{\frac{3 \gamma_{1} k_{4} \operatorname{sech}^{2}\left(\xi \sqrt{-\frac{k_{1} k_{4}}{k_{1} k_{3}-\beta_{4} v^{4} k_{2}}}\right)}{36 \gamma_{1}^{2}+\left[2 \gamma_{2} k_{4}\left(1+\epsilon \tanh \left(\xi \sqrt{-\frac{k_{1} k_{4}}{k_{1} k_{3}-\beta_{4} v^{4} k_{2}}}\right)\right)^{2}\right]}\right\}^{\frac{1}{2}},
$$

and the singular soliton solutions: 


$$
\varphi(\xi)=\left\{\frac{-3 \gamma_{1} k_{4} \operatorname{csch}^{2}\left(\xi \sqrt{-\frac{k_{1} k_{4}}{k_{1} k_{3}-\beta_{4} v^{4} k_{2}}}\right)}{36 \gamma_{1}^{2}+\left[2 \gamma_{2} k_{4}\left(1+\epsilon \operatorname{coth}\left(\xi \sqrt{-\frac{k_{1} k_{4}}{k_{1} k_{3}-\beta_{4} v^{4} k_{2}}}\right)\right)^{2}\right]}\right\}^{\frac{1}{2}},
$$

where satisfy the constraint condition: $\gamma_{1} k_{4}<0, \gamma_{2} k_{4}>0, \frac{k_{1} k_{4}}{k_{1} k_{3}-\beta_{4} v^{4} k_{2}}<0$, $\epsilon_{1}=0, \epsilon= \pm 1$, and $\xi=p Z-v t$.

5) If $\alpha>0, \gamma>0, \delta=0$, Then, we derive from Equation (19) and Equation (20) that Equation (51) has the solitary wave solutions:

$$
\varphi(\xi)=\left\{\frac{k_{4} \operatorname{sech}^{2}\left(\xi \sqrt{-\frac{k_{1} k_{4}}{k_{1} k_{3}-\beta_{4} v^{4} k_{2}}}\right)}{12 \gamma_{1}+4 \epsilon \sqrt{-2 \gamma_{2} k_{4}} \tanh \left(\xi \sqrt{-\frac{k_{1} k_{4}}{k_{1} k_{3}-\beta_{4} v^{4} k_{2}}}\right)}\right\}^{\frac{1}{2}},
$$

and

$$
\varphi(\xi)=\left\{\frac{-k_{4} \operatorname{csch}^{2}\left(\xi \sqrt{-\frac{k_{1} k_{4}}{k_{1} k_{3}-\beta_{4} v^{4} k_{2}}}\right)}{12 \gamma_{1}+4 \epsilon \sqrt{-2 \gamma_{2} k_{4}} \operatorname{coth}\left(\xi \sqrt{-\frac{k_{1} k_{4}}{k_{1} k_{3}-\beta_{4} v^{4} k_{2}}}\right)}\right\}^{\frac{1}{2}},
$$

where the Equation (60) satisfy the constraint condition: $\gamma_{1}>0, k_{4}>0$, $\gamma_{2} k_{4}<0, \frac{k_{1} k_{4}}{k_{1} k_{3}-\beta_{4} v^{4} k_{2}}<0, \epsilon_{1}=0, \epsilon= \pm 1$, and $\xi=p Z-v t$.

where the Equation (61) satisfy the constraint condition: $\gamma_{1}>0, k_{4}<0$, $\gamma_{2} k_{4}<0, \frac{k_{1} k_{4}}{k_{1} k_{3}-\beta_{4} v^{4} k_{2}}<0, \epsilon_{1}=0, \epsilon= \pm 1$, and $\xi=p Z-v t$.

6) If $\alpha<0, \gamma>0, \delta=0$, Then, we derive from Equation (21) and Equation (22) that Equation (51) has the periodic solutions:

$$
\varphi(\xi)=\left\{\frac{k_{4} \sec ^{2}\left(\xi \sqrt{\frac{k_{1} k_{4}}{k_{1} k_{3}-\beta_{4} v^{4} k_{2}}}\right)}{12 \gamma_{1}+4 \epsilon \sqrt{2 \gamma_{2} k_{4}} \tan \left(\xi \sqrt{\frac{k_{1} k_{4}}{k_{1} k_{3}-\beta_{4} v^{4} k_{2}}}\right)}\right\}^{\frac{1}{2}},
$$

and

$$
\varphi(\xi)=\left\{\frac{k_{4} \csc ^{2}\left(\xi \sqrt{\frac{k_{1} k_{4}}{k_{1} k_{3}-\beta_{4} v^{4} k_{2}}}\right)}{12 \gamma_{1}+4 \epsilon \sqrt{2 \gamma_{2} k_{4}} \cot \left(\xi \sqrt{\frac{k_{1} k_{4}}{k_{1} k_{3}-\beta_{4} v^{4} k_{2}}}\right)}\right\}^{\frac{1}{2}},
$$

where satisfy the constraint condition: $\gamma_{1}>0, k_{4}>0, \gamma_{2} k_{4}>0$, 
$\frac{k_{1} k_{4}}{k_{1} k_{3}-\beta_{4} v^{4} k_{2}}>0, \epsilon_{1}=0, \epsilon= \pm 1$, and $\xi=p Z-v t$.

7) If $\alpha>0, Q>0, \delta=0$, Then, we derive from Equation (23) and Equation (24) that Equation (51) has the bright soliton solutions:

$$
\varphi(\xi)=\left\{\frac{-k_{4} \operatorname{sech}\left(2 \xi \sqrt{-\frac{k_{1} k_{4}}{k_{1} k_{3}-\beta_{4} v^{4} k_{2}}}\right)}{2 \sqrt{9 \gamma_{1}^{2}+2 \gamma_{2} k_{4}}-6 \epsilon \gamma_{1} \operatorname{sech}\left(2 \xi \sqrt{-\frac{k_{1} k_{4}}{k_{1} k_{3}-\beta_{4} v^{4} k_{2}}}\right)}\right\}^{\frac{1}{2}},
$$

where satisfy the constraint condition: $k_{4}<0, \gamma_{2} k_{4}>0, \frac{k_{1} k_{4}}{k_{1} k_{3}-\beta_{4} v^{4} k_{2}}<0$, $\epsilon_{1}=0, \epsilon= \pm 1$, and $\xi=p Z-v t$.

8) If $\alpha>0, \beta<0, \gamma<0, Q>0, \delta=0$, Then, we derive from Equation (25) and Equation (26) that Equation (51) has the bright soliton solutions:

$$
\varphi(\xi)=\left\{\frac{-k_{4} \operatorname{sech}^{2}\left(\epsilon \xi \sqrt{-\frac{k_{1} k_{4}}{k_{1} k_{3}-\beta_{4} v^{4} k_{2}}}\right)}{4 \sqrt{9 \gamma_{1}^{2}+2 \gamma_{2} k_{4}}-\left[2 \sqrt{9 \gamma_{1}^{2}+2 \gamma_{2} k_{4}}+6 \gamma_{1}\right] \operatorname{sech}^{2}\left(\epsilon \xi \sqrt{-\frac{k_{1} k_{4}}{k_{1} k_{3}-\beta_{4} v^{4} k_{2}}}\right)}\right\}^{\frac{1}{2}},
$$

and the singular soliton solutions:

$$
\varphi(\xi)=\left\{\frac{-k_{4} \operatorname{csch}^{2}\left(\epsilon \xi \sqrt{-\frac{k_{1} k_{4}}{k_{1} k_{3}-\beta_{4} v^{4} k_{2}}}\right)}{4 \sqrt{9 \gamma_{1}^{2}+2 \gamma_{2} k_{4}}+\left[2 \sqrt{9 \gamma_{1}^{2}+2 \gamma_{2} k_{4}}-6 \gamma_{1}\right] \operatorname{csch}^{2}\left(\epsilon \xi \sqrt{-\frac{k_{1} k_{4}}{k_{1} k_{3}-\beta_{4} v^{4} k_{2}}}\right)},\right.
$$

where satisfy the constraint condition: $k_{4}<0, \gamma_{2} k_{4}>0, \frac{k_{1} k_{4}}{k_{1} k_{3}-\beta_{4} v^{4} k_{2}}<0$, $\epsilon_{1}=0, \epsilon= \pm 1$, and $\xi=p Z-v t$.

9) If $\alpha<0, \beta>0, \gamma<0, Q>0, \delta=0$, Then, we derive from Equation (27) and Equation (28) that Equation (51) has the periodic solutions:

$$
\varphi(\xi)=\left\{\frac{k_{4} \sec ^{2}\left(\epsilon \xi \sqrt{\frac{k_{1} k_{4}}{k_{1} k_{3}-\beta_{4} v^{4} k_{2}}}\right)}{4 \sqrt{9 \gamma_{1}^{2}+2 \gamma_{2} k_{4}}-\left[2 \sqrt{9 \gamma_{1}^{2}+2 \gamma_{2} k_{4}}-6 \gamma_{1}\right] \sec ^{2}\left(\epsilon \xi \sqrt{\frac{k_{1} k_{4}}{k_{1} k_{3}-\beta_{4} v^{4} k_{2}}}\right)}\right\}^{\frac{1}{2}},
$$

and

$$
\varphi(\xi)=\left\{\frac{k_{4} \csc ^{2}\left(\epsilon \xi \sqrt{\frac{k_{1} k_{4}}{k_{1} k_{3}-\beta_{4} v^{4} k_{2}}}\right)}{4 \sqrt{9 \gamma_{1}^{2}+2 \gamma_{2} k_{4}}-\left[2 \sqrt{9 \gamma_{1}^{2}+2 \gamma_{2} k_{4}}+6 \gamma_{1}\right] \csc ^{2}\left(\epsilon \xi \sqrt{\frac{k_{1} k_{4}}{k_{1} k_{3}-\beta_{4} v^{4} k_{2}}}\right)}\right\}^{\frac{1}{2}},
$$


where satisfy the constraint condition: $k_{4}>0, \gamma_{2} k_{4}>0, \frac{k_{1} k_{4}}{k_{1} k_{3}-\beta_{4} v^{4} k_{2}}>0$, $\epsilon_{1}=0, \epsilon= \pm 1$, and $\xi=p Z-v t$.

10) If $\alpha<0, Q>0, \delta=0$, Then, we derive from Equation (29) and Equation (30) that Equation (51) has the periodic solutions:

$$
\varphi(\xi)=\left\{\frac{-k_{4} \sec \left(2 \xi \sqrt{\frac{k_{1} k_{4}}{k_{1} k_{3}-\beta_{4} v^{4} k_{2}}}\right)}{2 \sqrt{9 \gamma_{1}^{2}+2 \gamma_{2} k_{4}}-6 \epsilon \gamma_{1} \sec \left(2 \xi \sqrt{\frac{k_{1} k_{4}}{k_{1} k_{3}-\beta_{4} v^{4} k_{2}}}\right)}\right\}^{\frac{1}{2}},
$$

and

$$
\varphi(\xi)=\left\{\frac{-k_{4} \csc \left(2 \xi \sqrt{\frac{k_{1} k_{4}}{k_{1} k_{3}-\beta_{4} v^{4} k_{2}}}\right)}{2 \sqrt{9 \gamma_{1}^{2}+2 \gamma_{2} k_{4}}-6 \epsilon \gamma_{1} k_{1} \csc \left(2 \xi \sqrt{\frac{k_{4}}{k_{1} k_{3}-\beta_{4} v^{4} k_{2}}}\right)}\right\}^{\frac{1}{2}},
$$

where satisfy the constraint condition: $k_{4}<0, \gamma_{2} k_{4}>0, \frac{k_{1} k_{4}}{k_{1} k_{3}-\beta_{4} v^{4} k_{2}}>0$, $\epsilon_{1}=0, \epsilon= \pm 1$, and $\xi=p Z-v t$.

\section{Conclusion}

The special kind of $\left(G^{\prime} / G\right)$-expansion, the new mapping method successfully solved the higher order dispersion nonlinear schrodinger equation and the generalized nonlinear schrodinger equation, and new exact travelling wave solutions are obtained. It includes the solitary wave solutions, singular soliton solutions, bright and dark soliton solutions and periodic solutions. Compared with other methods, it is an effective method to solve the exact traveling wave solution, therefore, this method can be extended to solve other nonlinear PDEs.

\section{Conflicts of Interest}

The authors declare no conflicts of interest regarding the publication of this paper.

\section{References}

[1] Feng, Z.S. (2002) The First Integral Method to Study the Burgers-Korteweg-de Vries Equation. Journal of Physics A: Mathematical and General, 35, 343-349. https://doi.org/10.1088/0305-4470/35/2/312

[2] Liu, S., Fu, Z., Liu, S.D. and Zhao, Q. (2001) Jacobi Elliptic Function Expansion Method and Periodic Wave Solutions of Nonlinear Wave Equations. Physics Letters $A$, 289, 69-74. https://doi.org/10.1016/S0375-9601(01)00580-1

[3] Zhang, S. (2006) The Periodic Wave Solutions for the (2+1) Dimensional Konopelchenko Dubrovsky Equations. Chaos, Solitons \& Fractals, 30, 1213-1220. https://doi.org/10.1016/j.chaos.2005.08.201 
[4] He, J.H. and Zhang, L.N. (2008) Generalized Solitary Solution and Compacton-Like Solution of the Jaulent-Miodek Equations Using the Exp-Function Method. Physics Letters A, 372, 1044-1047. https://doi.org/10.1016/j.physleta.2007.08.059

[5] Kim, H., Bae, J.H. and Sakthivel, R. (2014) Exact Travelling Wave Solutions of Two Important Nonlinear Partial Differential Equations. Zeitschrift für Naturforschung $A, 69,155-162$. https://doi.org/10.5560/zna.2014-0001

[6] Zhang, J., Jiang, F. and Zhao, X. (2010) An Improved $\left(G^{\prime} / G\right)$-Expansion Method for Solving Nonlinear Evolution Equations. International Journal of Computer Mathematics, 87, 1716-1725. https://doi.org/10.1080/00207160802450166

[7] Wazwaz, A.M. (2007) Traveling Wave Solutions to (2+1)-Dimensional Nonlinear Evolution Equations. Journal of Natural Sciences and Mathematics, 1, 1-13.

[8] Ma, W.X. (1993) Travelling Wave Solutions to a Seventh Order Generalized KdV Equation. Physics Letters A, 180, 221-224. https://doi.org/10.1016/0375-9601(93)90699-Z

[9] Conte, R. and Musette, M. (1992) Link between Solitary Waves and Projective Riccati Equations. Journal of Physics A, 25, 5609-5623. https://doi.org/10.1088/0305-4470/25/21/019

[10] Kudryashov, N.A. (1991) On Types of Nonlinear Nonintegrable Differential Equations with Exact Solutions. Physics Letters A, 155, 269-275. https://doi.org/10.1016/0375-9601(91)90481-M

[11] Wazwaz, A.M. (2004) A Sine-Cosine Method for Handling Nonlinear Wave Equations. Mathematical and Computer Modelling, 40, 499-508. https://doi.org/10.1016/j.mcm.2003.12.010

[12] Wang, D.S. (2009) A Systematic Method to Construct Hirotas Transformations of Continuous Soliton Equations and Its Applications. Computers and Mathematics with Applications, 58, 146-153. https://doi.org/10.1016/j.camwa.2009.03.077

[13] Li, Y., Shan, W.R., Shuai, T.P. and Rao, K. (2015) Bifurcation Analysis and Solutions of a Higher-Order Nonlinear Schrödinger Equation. Mathematical Problems in Engineering, 2015, Article ID: 408586. https://doi.org/10.1155/2015/408586

[14] Huang, Y. and Liu, P. (2014) New Exact Solutions for a Class of High-Order Dispersive Cubic-Quintic Nonlinear Schröinger Equation. Canadian Center of Science and Education, Richmond Hill. https://doi.org/10.5539/jmr.v6n4p104

[15] Geng, Y.X. and Li, J.B. (2010) Exact Explicit Traveling Wave Solutions for Two Nonlinear Schrodinger Type Equations. Applied Mathematics and Computation, 217, 1509-1521. https://doi.org/10.1016/j.amc.2009.06.031

[16] Kudryashov, N.A. (2010) A Note on the $\left(G^{\prime} / G\right)$-Expansion Method. Applied Mathematics and Computation, 217, 1755-1758. https://doi.org/10.1016/j.amc.2010.03.071

[17] Alam, M.N., Akbar, M.A. and Mohyud-Din, S.T. (2014) A Novel (G'/G)-Expansion Method and Its Application to the Boussinesq Equation. Chinese Physics B, 23, Article ID: 020203. https://doi.org/10.1088/1674-1056/23/2/020203

[18] Alam, M.N. and Akbar, M.A. (2014) Traveling Wave Solutions of the Nonlinear $(1+1)$-Dimensional Modified Benjamin-Bona-Mahony Equation by Using Novel $\left(G^{\prime} / G\right)$-Expansion Method. Physical Review \& Research International, 4, 147-165.

[19] Zeng, X. and Yong, X. (2008) A New Mapping Method and Its Applications to Nonlinear Partial Differential Equations. Physics Letters A, 372, 2225-2228. https://doi.org/10.1016/j.physleta.2008.09.025 
[20] Zayed, E.M.E. and Al-Nowehy, A.G. (2017) Many New Exact Solutions to the Higher-Order Nonlinear Schrödinger Equation with Dervative Non-Kerr Nonlinear Term. Optik-International Journal for Light and Electron Optics, 143, 84-103. https://doi.org/10.1016/j.ijleo.2017.06.025

[21] Zayed, E.M.E. and Alurrfi, K.A.E. (2017) Solitons and Other Solutions for Two Nonlinear Schrödinger Equations Using the New Mapping Method. Optik-International Journal for Light and Electron Optics, 144, 132-148. https://doi.org/10.1016/j.ijleo.2017.06.101

[22] Zayed, E.M.E. and Al Nowehy, A.G. (2017) Exact Solutions and Optical Soliton Solutions for the Nonlinear Schrödinger Equation with Fourth-Order Dispersion and Cubic-Quintic Nonlinearity. Ricerche di Matematica, 66, 531-552. https://doi.org/10.1007/s11587-017-0319-y

[23] Arshad, M., Seadawy, A.R. and Lu, D. (2017) Elliptic Function and Solitary Wave Solutions of the Higher-Order Nonlinear Schrödinger Dynamical Equation with Fourth-Order Dispersion and Cubic-Quintic Nonlinearity and Its Stability. European Physical Journal Plus, 132, Article No. 371. https://doi.org/10.1140/epjp/i2017-11655-9

[24] Zayed, E.M.E. and Al Nowehy, A.G. (2017) Jacobi Elliptic Solutions, Solitons and Other Solutions for the Nonlinear Schrödinger Equation with Fourth-Order Dispersion and Cubic-Quintic Nonlinearity. European Physical Journal Plus, 132, Article No. 475. https://doi.org/10.1140/epjp/i2017-11752-9

[25] Arshad, M., Seadawy, A.R. and Lu, D. (2017) Exact Bright Dark Solitary Wave Solutions of the Higher-Order Cubic Quintic Nonlinear Schrödinger Equation and Its Stability. Optik-International Journal for Light and Electron Optics, 138, 40-49. https://doi.org/10.1016/j.ijleo.2017.03.005

[26] Davydova, T.A. and Zaliznyak, Y.A. (2001) Schrödinger Ordinary Solitons and Chirped Solitons: Fourth-Order Dispersive Effects and Cubic-Quintic Nonlinearity. Physica D Nonlinear Phenomena, 156, 260-282. https://doi.org/10.1016/S0167-2789(01)00269-X

[27] Huang, D.J., Li, D.S. and Zhang, H.Q. (2007) Explicit and Exact Travelling Wave Solutions for the Generalized Derivative Schrodinger Equation. Chaos Solitons and Fractals, 31, 586-593. https://doi.org/10.1016/j.chaos.2005.10.007

[28] Liu, X. and Simpson, G. (2013) Stability of Solitary Waves for a Generalized Derivative Nonlinear Schrodinger Equation. Journal of Nonlinear Science, 23, 557-583. https://doi.org/10.1007/s00332-012-9161-2

[29] Xing, L., Ma, W.X., Yu, J. and Khalique, C.M. (2016) Solitary Waves with the Madelung Fluid Description: A Generalized Derivative Nonlinear Schrodinger Equation. Communications in Nonlinear Science and Numerical Simulation, 31, 40-46. https://doi.org/10.1016/j.cnsns.2015.07.007

[30] Tian, S., Zhang, T. and Zhang, H. (2009) Darboux Transformation and New Periodic Wave Solutions of Generalized Derivative Nonlinear Schrodinger Equation. Physica Scripta, 80, Article ID: 065013. https://doi.org/10.1088/0031-8949/80/06/065013

[31] Zhang, H. (2009) New Application of the $\left(G^{\prime} / G\right)$-Expansion Method? Communications in Nonlinear Science and Numerical Simulation, 14, 3220-3225. https://doi.org/10.1016/j.cnsns.2009.01.006

[32] Zayed, E.M.E. and El Mailky, M.A.S. (2011) The $\left(G^{\prime} / G\right)$-Expansion Method for Solving Nonlinear Klein-Goedon Equations. AIP Conference Proceedings, 1389, 2020-2024. https://doi.org/10.1063/1.3637012 
[33] Zayed, E.M.E. (2010) Equivalence of the $\left(G^{\prime} / G\right)$-Expansion Method and the Tanh-Coth Function Method. AIP Conference Proceedings, 1281, 2225-2228. https://doi.org/10.1063/1.3498417

[34] Zeng, X. and Yong, X. (2008) A New Mapping Method and Its Applications to Nonlinear Partial Differential Equations. Physics Letters A, 372, 6602-6607. https://doi.org/10.1016/j.physleta.2008.09.025 